\title{
Oleanolic acid alleviates diabetic rat carotid artery injury through the inhibition of NLRP3 inflammasome signaling pathways
}

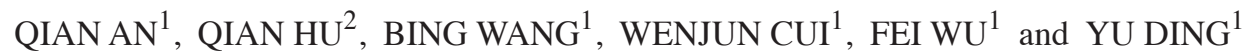 \\ ${ }^{1}$ Department of Vascular Surgery, The Fifth Affiliated Hospital of Zhengzhou University, Zhengzhou, Henan 470000; \\ ${ }^{2}$ Staff Room of Surgery, Zhengzhou Railway Vocational Technical College, Zhengzhou, Henan 450052, P.R. China
}

Received July 28, 2016; Accepted July 17, 2017

DOI: $10.3892 / \mathrm{mmr} .2017 .7594$

\begin{abstract}
The overexpression of inflammasome components is correlated with diabetes-associated complications. Oleanolic acid is a triterpenoid compound which is important in arterial injury. The present study evaluated whether oleanolic acid improved diabetic rat carotid artery injury through the inhibition of nucleotide-binding domain, leucine-rich-containing family, pyrin domain-containing-3 (NLRP3) inflammasomes signaling pathways. A diabetic rat model was induced using streptozotocin $(60 \mathrm{mg} / \mathrm{kg})$ and underwent carotid artery injury. Morphometric analysis was performed using hematoxylin and eosin staining. The mRNA and protein levels were assayed by reverse transcription-quantitative polymerase chain reaction and western blotting, respectively. It was found that oleanolic acid (100 mg/kg/day) improved body weight, glucose metabolic disorders, neointimal hyperplasia and endothelial dysfunction in diabetic rats with carotid artery injury. In addition, oleanolic acid administration significantly downregulated the mRNA and protein expression levels of endothelin 1 in diabetic rats. Oleanolic acid decreased the intimal area and the ratio of neointima to media in diabetic rats. Serum levels of tumor necrosis factor- $\alpha$, interleukin (IL)-1 $\beta$, IL- 6 and IL-18 in the oleanolic acid-treated diabetic rats were downregulated. Consistent with the serum results, it was demonstrated that oleanolic acid administration caused a significant decrease in the levels of NLRP3, caspase-1 and IL- $1 \beta$ in the carotid arteries of diabetic rats. Taken together, these observations suggested that oleanolic acid attenuated carotid artery injury in diabetic rats and the underlying mechanism was mediated, at least partially, through the suppression of NLRP3 inflammasome signaling pathways.
\end{abstract}

Correspondence to: Dr Bing Wang, Department of Vascular Surgery, The Fifth Affiliated Hospital of Zhengzhou University, 3 Kangfuqianjie Road, Zhengzhou, Henan 470000, P.R. China E-mail: wb_fhu@163.com

Key words: oleanolic acid, nucleotide-binding domain, leucine-rich-containing family, pyrin domain-containing-3, diabetes, neointimal hyperplasia, carotid artery injury

\section{Introduction}

Diabetes mellitus (DM) is a significant risk factor for carotid arterial injury by carotid intima-media thickness (1). Compared with patients without diabetes, patients with diabetes often exhibit severe neointimal hyperplasia (2). Hyperglycemia can also induce neointimal hyperplasia following vascular injury in a rat carotid artery injury model $(3,4)$. Inflammation is important in this process, provoking vascular smooth muscle cells (VSMCs) to migrate to the intima, which results in neointimal formation (5). Increasing evidence indicates that inflammatory cytokines are implicated in peripheral arterial disease and carotid artery disease (6). Nucleotide-binding domain, leucine-rich-containing family, pyrin domain-containing-3 (NLRP3) inflammasome components are abundantly expressed in microvascular endothelial cells, and the activation of NLRP3 inflammasome signaling enhances neointimal formation in mice (7). The NLRP3 inflammasome, as intracellular inflammatory machinery, has been reported to be involved in obesity-associated coronary atherosclerotic injury and endothelial dysfunction (7). Clinical studies have shown that NLRP3 is overexpressed in the aorta of patients with coronary atherosclerosis and carotid atherosclerotic plaques $(8,9)$. These findings suggest that the NLRP3 inflammasome mechanism may be involved in hyperglycemia-induced carotid arterial injury.

Oleanolic acid is a triterpenoid compound, which exists widely in food and Chinese herbal medicine (10). It has a variety of biological effects, including antioxidant (10), renoprotective (11), hepatoprotective (12), and anticancer effects (13). In addition, oleanolic acid, as a synergistic therapeutic drug, improves glucose and insulin homeostasis in $\mathrm{db} / \mathrm{db}$ diabetic mice (14). Oleanolic acid, or its isomer, can improve type 2 diabetes-associated complications, particularly inflammation, through inhibition of the nuclear transcription factor- $\kappa \mathrm{B}(\mathrm{NF}-\kappa \mathrm{B})$ signaling pathway (15). Oleanolic acid suppresses NF- $\kappa B$ signaling by inhibiting the lipopolysaccharide (LPS)-induced phosphorylation of inhibitor of NF- $\mathrm{kB}(15,16)$. The administration of ursolic acid, an isomer of oleanolic acid, in mice fed a high fat diet (HFD) also inhibits the expression of inflammatory cytokines tumor necrosis factor (TNF)- $\alpha$ and interleukin (IL)-1 through NF- $\mathrm{kB}$ signaling (16). The anti-atherogenic effects of oleanolic acid in apolipoprotein E-knockout mice are mediated by reducing the 
expression of inducible nitric oxide synthase (17). However, the arterial protection of oleanolic acid in diabetic rats by NLRP3 inflammasome signaling remains to be fully elucidated.

In the present study, whether the long-term administration of oleanolic acid can protect against hyperglycemia-induced carotid artery injury was investigated in a diabetic rat animal model. The expression levels of NLRP3 inflammasome components were also measured in the carotid arteries of diabetic rats.

\section{Materials and methods}

Animal experiments. Male Sprague-Dawley (SD) rats $(\mathrm{n}=18$; 8-week-old) were purchased from the Animal Center of Zhengzhou University (Zhengzhou, China) and fed in an SPF laboratory. The rats were fed under controlled temperature $\left(23 \pm 2^{\circ} \mathrm{C}\right)$ and humidity $(55 \pm 5 \%)$ with an artificial 12 -h light/dark cycle, and were given free access to food and tap water. The rats were allowed to acclimate to the environment for 1 week. All experimental procedures were performed in accordance with the guidelines on Animal Care of the Fifth Affiliated Hospital of Zhengzhou University (Zhengzhou, China). Rats weighing 200-220 g were used for the experiments in the present study, which were randomized into three groups: i) control group $(n=6)$; ii) diabetic rats with carotid artery injury (model group, $n=6$ ); and iii) diabetic rats with carotid artery injury + oleanolic acid (HPLC $\geq 98 \%$; Aladdin Chemical Co., Ltd., Shanghai, China) treatment (oleanolic acid group, $n=6$ ). The establishment of a streptozotocin (STZ)-induced diabetic rat model with carotid artery injury was performed as described previously (3). All other chemicals were of analytical grade and purchased from Sigma-Aldrich (Sigma-Aldrich; Merck KGaA, Darmstadt, Germany).

Histological examination. The normal or injured carotid arteries from the rats were fixed in $4 \%$ paraformaldehyde for 1 week and then embedded in paraffin. Sections measuring $5 \mu \mathrm{m}$ were cut and stained with hematoxylin \& eosin (H\&E). For morphologic analysis of neointimal formation, Image-Pro Plus 5.0 image analysis software (Media Cybernetics Inc., Rockville, MD, USA) was used. The medial and intimal cross-sectional areas were measured, and the intima/media ratios were calculated.

Serum levels of inflammatory cytokines. Following sacrifice of the rats, blood was collected using Vacutainer tubes (BD Biosciences, Franklin Lakes, NJ, USA) and centrifuged immediately at $3,000 \mathrm{x}$ for $10 \mathrm{~min}$ at $4^{\circ} \mathrm{C}$. The supernatant was then collected and stored at $-80^{\circ} \mathrm{C}$ until used for the subsequent assay. The levels of TNF- $\alpha$, IL-1 $\beta$, IL- 6 and IL-18 were analyzed using enzyme-linked immunosorbent assay (ELISA) kits (Biosource, Camarillo, CA, USA) according to the manufacturer's instructions. ELISA kits were used to measure the levels of endothelin 1 (ET-1; cat. no. E-EL-R0167; Elabscience Biotechnology Co., Ltd., Wuhan, China), nitric oxide (NO; cat. no. A012-1; Nanjing Jiancheng Biology Engineering Institute, Nanjing, China) and von Willebrand factor (vWF; cat. no. E-EL-R1079; Elabscience Biotechnology) in the supernatant on an ELISA reader (BioTek Instruments, Inc., Winooski, VT, USA) according to the manufacturers' instructions.
Vascular permeability assay. Aortic blood vessel leakage was quantitated using Evans blue dye, which binds non-covalently to plasma albumin in the blood stream, as described previously (18). Aortic blood vessels were visualized under a microscope (Leica DM 2500; Leica Microsystems GmbH, Wetzlar, Germany).

Reverse transcription-quantitative polymerase chain reaction $(R T-q P C R)$ analysis. RNA was extracted using TRIzol ${ }^{\circledR}$ (Invitrogen; Thermo Fisher Scientific, Inc., Waltham, MA, USA) according to the manufacturer's instructions. The synthesis of cDNA was performed with $2 \mu \mathrm{g}$ total RNA using Moloney murine leukemia virus reverse transcriptase (Promega Corporation, Madison, WI, USA) and oligo(dT)15 primers (Thermo Fisher Scientific, Inc.) according to the manufacturer's instructions. The qPCR was performed using an Applied Biosystems 7300 Real-Time PCR system (Thermo Fisher Scientific, Inc.). Reaction mixtures $(25 \mu \mathrm{l})$ were prepared as follows: $12.5 \mu \mathrm{l} \mathrm{SYBR-Green} \mathrm{Supermix}$ (Bio-Rad Laboratories, Inc., Hercules, CA, USA), $1 \mu \mathrm{l}$ cDNA, $300 \mathrm{nM}$ of each primer, and $\mathrm{DEPC} \mathrm{H}_{2} \mathrm{O}$ to a final volume of $25 \mu \mathrm{l}$. Thermocycling procedure was performed as follows: $94^{\circ} \mathrm{C}$ for $1 \mathrm{~min}, 40 \mathrm{cycles}$ of $94^{\circ} \mathrm{C}$ for $30 \mathrm{sec}, 50^{\circ} \mathrm{C}$ for $30 \mathrm{sec}$ and $72^{\circ} \mathrm{C}$ for $30 \mathrm{sec}$. The quantification cycle fluorescence value $(\mathrm{Cq})$ was calculated using SDS software, version 2.1 (Applied Biosystems; Thermo Fisher Scientific, Inc.), and the relative mRNA expression levels of RANK were calculated using the $2^{-\triangle \Delta C q}$ method (19) and normalized to the internal control, glyceraldehyde 3-phosphate dehydrogenase (GAPDH). The following primer sequences were used: ET-1 forward, 5'-AAGCGCTGTTCCTGTTCTTCA-3' and reverse, 5'-CTT GATGCTATTGCTGATGG-3'; NLRP3 forward, 5'-AAA GCCAAGAATCCACAGTGTAAC-3' and reverse, 5'-TTG CCTCGCAGGTAAAGGT-3'; capsase-1 forward, 5'-AGG CATGACAATGCTGCTACAA-3' and reverse, 5'-TGTGCA AATGCCTCCAGCTC-3'; IL-1 $\beta$ forward, 5'-TCGCCAGTG AAATGATGGCTTA-3' and reverse, 5'-GTCCATGGCCAC AACAACTGA-3'; GAPDH forward, 5'-ACAGGGGAGGTG ATAGCATT-3' and reverse, 5'-GACCAAAAGCCTTCATAC ATCTC-3'

Western blot analysis. The normal and injured carotid arteries of the rats were homogenized and lysed in NP-40 buffer (Beyotime Institute of Biotechnology, Haimen, China). Following 5-10 min of boiling, the cells were centrifuged at $10,000 \times \mathrm{g}$ at $4^{\circ} \mathrm{C}$ for $10 \mathrm{~min}$ to obtain the supernatant. Protein concentrations were determined using the Bicinchoninic Acid kit for Protein Determination (Sigma-Aldrich; Merck KGaA). Protein samples $(50 \mu \mathrm{g})$ were separated by $10 \%$ sodium dodecyl sulfate-polyacrylimide gel electrophoresis and transferred onto polyvinylidene difluoride membranes (EMD Millipore, Billerica, MA, USA). The membranes were blocked with $5 \%(\mathrm{w} / \mathrm{v})$ non-fat milk powder in Tris-buffered saline and $0.1 \%$ (w/v) with Tween-20, and incubated with the following primary antibodies: NLRP3 (cat. no. ab210491, 1:500; Abcam), ET-1 (cat. no. sc-57116, 1:10,00) caspase-1 (cat. no. sc-398715, 1:500), IL-1 $\beta$ (cat. no. sc-515598, 1:10,00) and $\beta$-actin (cat. no. sc-130065, 1:2,000) (all from Santa Cruz Biotechnology, Inc., Dallas, TX, USA) overnight at $4^{\circ} \mathrm{C}$. Following washing with PBS three times ( $5 \mathrm{~min} / \mathrm{each})$, the membranes were 

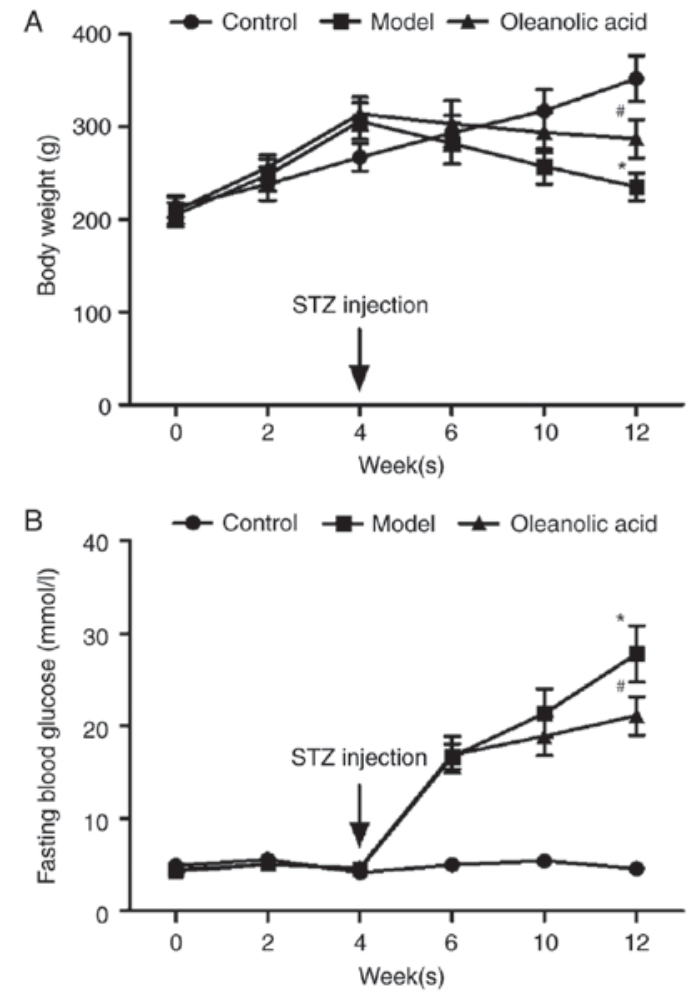

Figure 1. Oleanolic acid improves body weight and glucose levels. Normal rats and STZ-induced diabetic rats were fed with a high fat diet for 4 weeks prior to STZ injection. Subsequently, the normal rats and STZ-induced diabetic rats were fed with or without oleanolic acid $(100 \mathrm{mg} / \mathrm{kg} / \mathrm{d})$ between weeks 6 and 12. (A) Body weight and (B) fasting blood glucose were measured every 2 weeks during the experimental period. Values are expressed as the mean \pm standard error of the mean ( $n=6$ in each group). ${ }^{\#} \mathrm{P}<0.05$, vs. non-diabetic control group; ${ }^{*} \mathrm{P}<0.05$, vs. model group. STZ, streptozotocin.

incubated with HRP-conjugated anti-IgG (cat. no. sc-516102, 1:10,000; Santa Cruz Biotechnology, Inc.) at room temperature for $2 \mathrm{~h}$. Signal detection was performed using an ECL system (GE Healthcare Life Sciences, Chalfont, UK). Quantitative analysis of protein was assessed using Quantity One software version 4.5 (Bio Rad Laboratories, Inc.).

Statistical analysis. Data are expressed as the mean \pm standard error of the mean. All statistical analyses were performed using GraphPad Prism software, version 5.0 (GraphPad Software, Inc., La Jolla, CA, USA). Groups were compared using one-way analysis of variance, followed by Tukey's multiple comparison post hoc test to compare the mean values of each group. $\mathrm{P}<0.05$ was considered to indicate a statistically significant difference.

\section{Results}

Oleanolic acid improves body weight and glucose levels in rats with hyperglycemia-induced carotid artery injury. As shown in Fig. 1A and B, body weights steadily increased, and the fasting blood glucose (FBG) level was maintained within the normal range of 3.9-6.1 mmol/l in the non-diabetic control rats during the experimental period. In the rats fed with a HFD 4 weeks prior to STZ injection, the body weights of the rats significantly increased, however, injection of STZ markedly
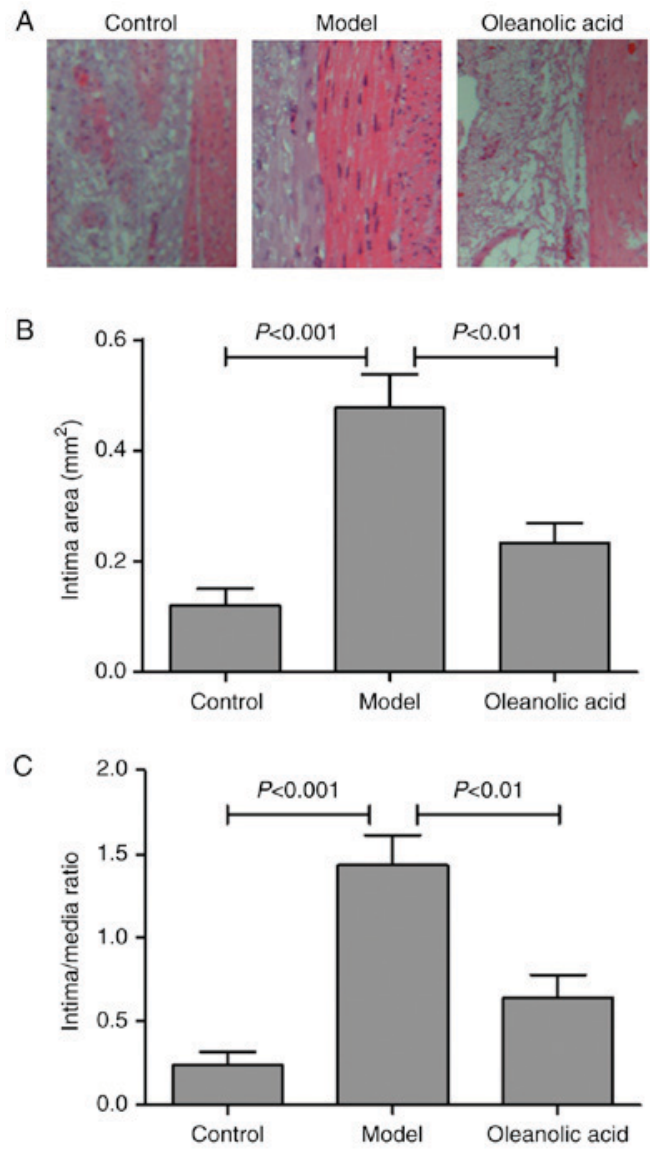

Figure 2. Oleanolic acid alleviates neointimal hyperplasia in the diabetic rat carotid artery injury model. (A) Morphologic analysis of neointimal hyperplasia was performed using hematoxylin and eosin staining (magnification, x200). (B) Quantification showed that oleanolic acid reduced the intima area. (C) Quantification of the ratio of intima area to media area supported the inhibitory effect of oleanolic acid on neointimal hyperplasia in the rats with hyperglycemia-induced carotid artery injury. Values are expressed as the mean \pm standard error of the mean ( $n=6$ in each group).

decreased the body weights of the rats (Fig. 1A). At 2 weeks post-STZ injection, the FBG values of the diabetic rats and oleanolic acid-treated diabetic rats increased from $4.6 \mathrm{mmol} / 1$ at week 4 to $16.7 \mathrm{mmol} / \mathrm{l}$ at week 6 , and $4.7 \mathrm{mmol} / \mathrm{l}$ at week 4 to $16.9 \mathrm{mmol} / \mathrm{l}$ at week 6 , respectively. These increases were significantly higher than that of the control group $(5.0 \mathrm{mmol} / \mathrm{l})$ at week 6 (Fig. 1B). Oleanolic acid administration reversed the HFD- and STZ-induced changes in body weight and glucose metabolic disorder (Fig. 1A and B).

Oleanolic acid alleviates carotid artery injury in diabetic rats. The degree of neointimal hyperplasia was evaluated by morphologic analysis with H\&E staining. The results demonstrated that the injured carotid arteries of the model group developed severe stenosis and neointimal hyperplasia, compared with the non-diabetic normal rats (Fig. 2A). Oleanolic acid treatment significantly reduced the intimal area, compared with that in the model group (Fig. 2B). The intima/media ratio was also lower in the oleanolic acid-treated group, compared with that in the model group (Fig. 2C).

Oleanolic acid improves endothelial function in rats with hyperglycemia-induced carotid artery injury. ET-1 is a potent 

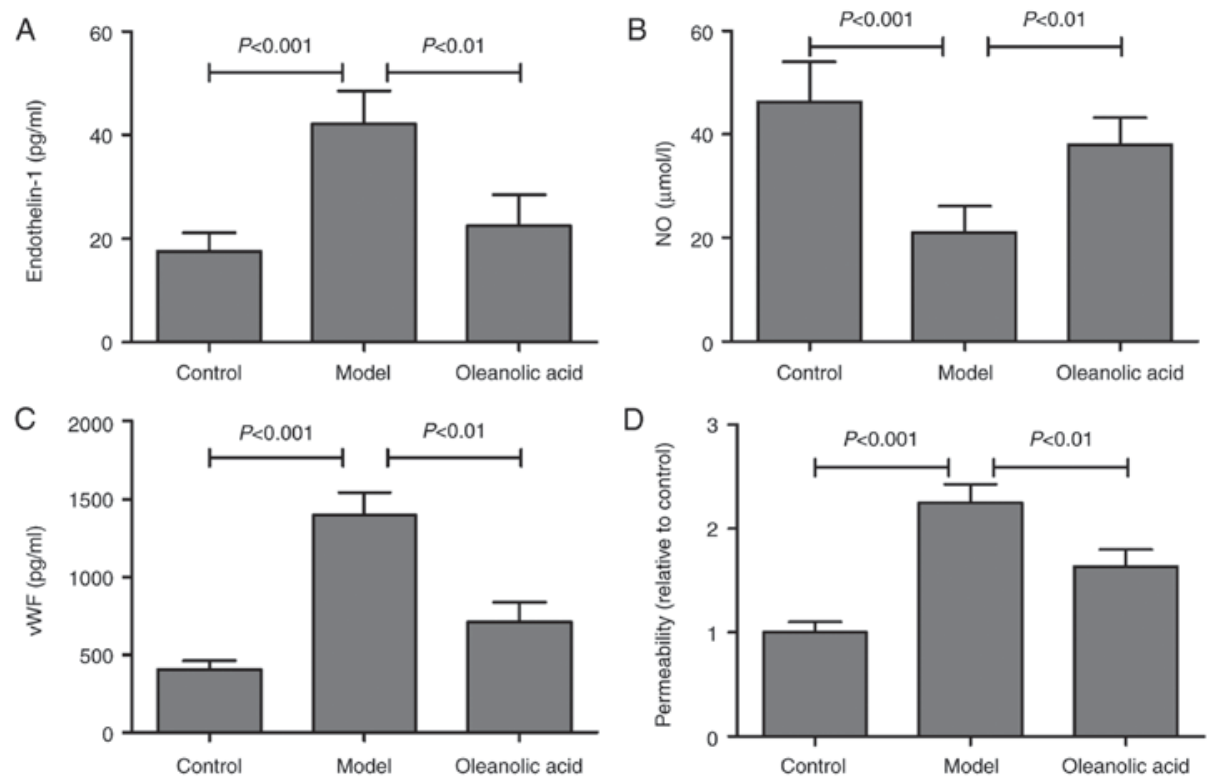

Figure 3. Oleanolic acid improves endothelial function in the diabetic rat carotid artery injury model. The serum levels of (A) ET-1, (B) NO and (C) vWF were measured using enzyme-linked immunosorbent assay kits. (D) Vascular permeability was assessed in the carotid artery by the presence of extravascular noncovalent albumin-bound Evans blue. Values are expressed as the mean \pm standard error of the mean ( $\mathrm{n}=6$ in each group). ET-1, endothelin 1; NO, nitric oxide; vWF, von Willebrand factor.

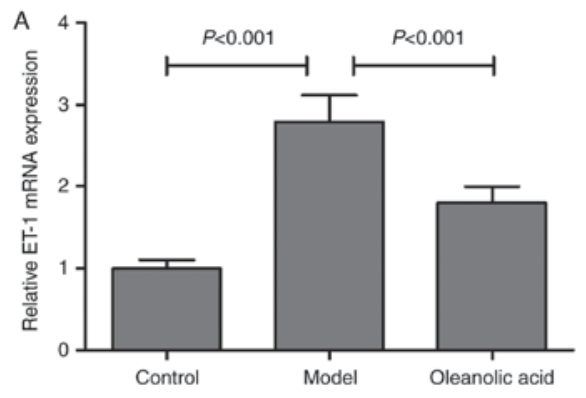

B

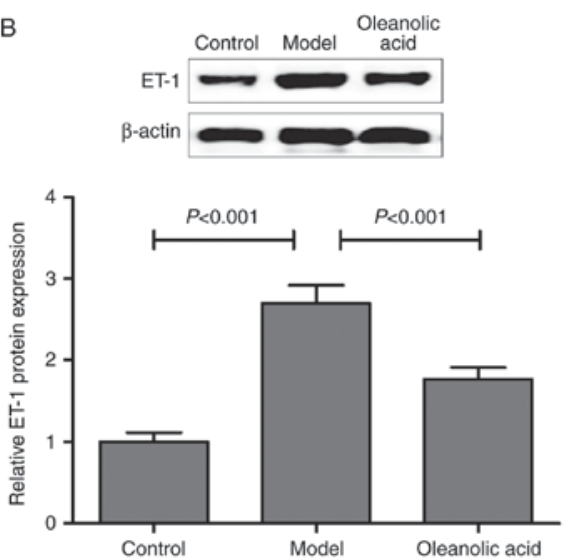

Figure 4. Oleanolic acid regulates the expression of ET-1. (A) mRNA and (B) protein expression of ET-1 were determined using reverse transcription-quantitative polymerase chain reaction and western blot analyses, respectively. Values are expressed as the mean \pm standard error of the mean $(n=6$ in each group). ET-1, endothelin 1.

vasoconstrictor, and its increase in endothelial cells leads to endothelial dysfunction and cardiovascular disorders (20). NO is the most important vascular relaxing factor, which is also regulated in the endothelium, and alterations in the endothelial production of NO are known to correlate with endothelial dysfunction (21). Compared with the normal rats, an increase in the serum level of ET-1 was observed in the model group, whereas oleanolic acid administration significantly downregulated the level of ET-1 in the diabetic rats (Fig. 3A). The results also showed that the levels of NO were significantly decreased in the serum of diabetic rats, compared with the non-diabetic control group. Oleanolic acid administration significantly prevented the hyperglycemia-induced decrease $\mathrm{NO}$ in diabetic rats (Fig. 3B). The serum concentration of vWF, a well-known marker of endothelial function/injury, was significantly higher in the diabetic rats, compared with that in the control group rats (Fig. 3C). Oleanolic acid treatment significantly reduced the levels of $\mathrm{vWF}$ in the diabetic rats. The findings also demonstrated that oleanolic acid significantly reversed the hyperglycemia-induced increase in vascular permeability (Fig. 3D), which is a characteristic of diabetic vasculopathy (22).

Consistent with the results described above, the present study confirmed that the mRNA and protein expression levels of ET-1 were markedly upregulated in the carotid artery of diabetic rats, compared with those of non-diabetic control rats. Oleanolic acid administration significantly downregulated the mRNA and protein expression of ET-1 in the diabetic rats (Fig. 4A and B).

Oleanolic acid inhibits NLRP3 inflammasome signaling in rats with hyperglycemia-induced carotid artery injury. As shown in Fig. 5A, hyperglycemia resulted in upregulation of the serum level of TNF- $\alpha$ in the rat model of carotid artery injury, compared with that in the control group. Similarly, the serum levels of IL-1 $\beta$ (Fig. 5B), IL-6 (Fig. 5C) and IL-18 (Fig. 5D) were significantly increased in the diabetic rats, compared with those in the control group rats. However, oleanolic acid administration led to significant decreases in the levels of TNF- $\alpha$, IL-1 $\beta$, IL-6 and IL-18 (Fig. 5A-D). These 

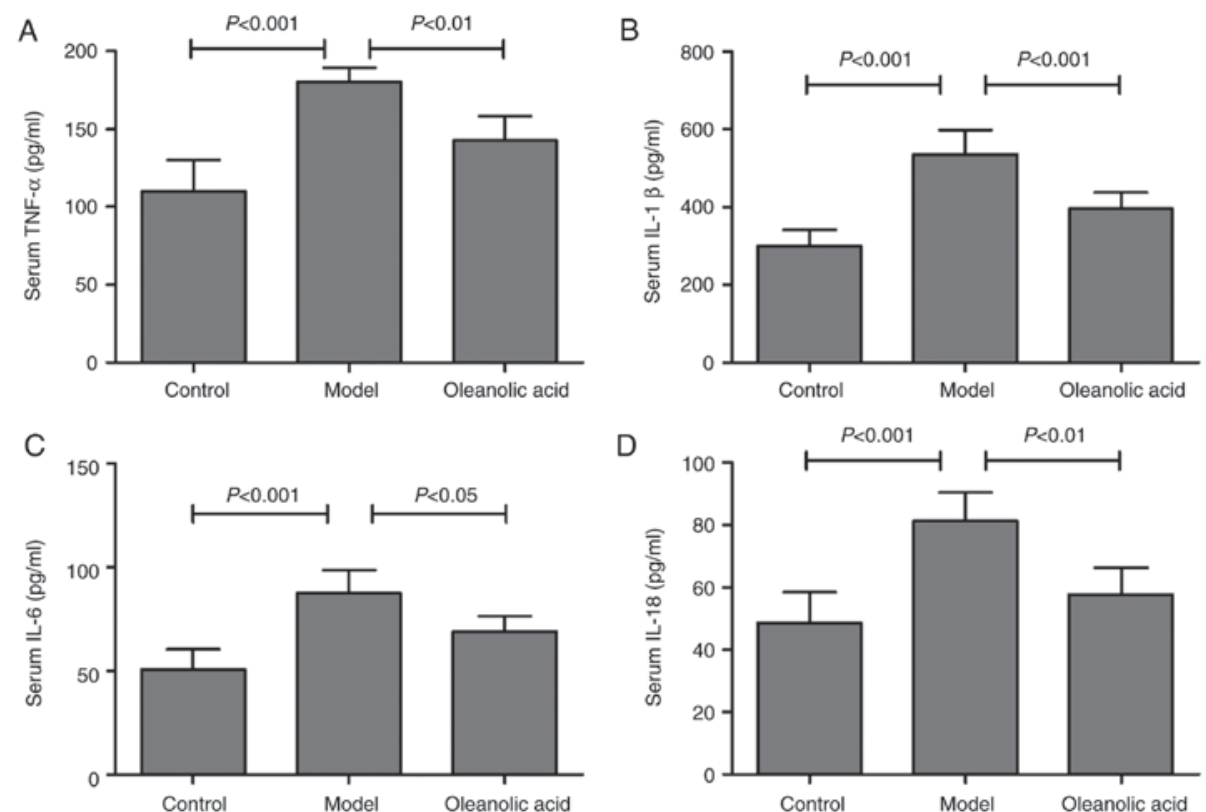

Figure 5. Oleanolic acid reduces levels of pro-inflammatory cytokines. Normal rats and streptozotocin-induced diabetic rats were fed with or without oleanolic acid (100 mg/kg/day) between weeks 6 and 12. The levels of (A) TNF- $\alpha$, (B) IL-1 $\beta$, (C) IL-6 and (D) IL-18 were analyzed using enzyme-linked immunosorbent assays. Values are expressed as the mean \pm standard error of the mean ( $\mathrm{n}=6$ in each group). ET-1, endothelin 1 ; TNF- $\alpha$, tumor necrosis factor- $\alpha$; IL, interleukin.

A

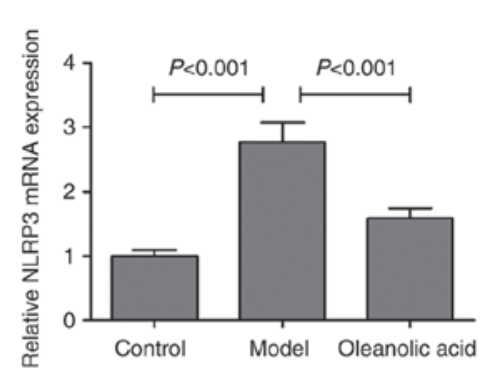

$\mathrm{D}$
$\mathrm{B}$

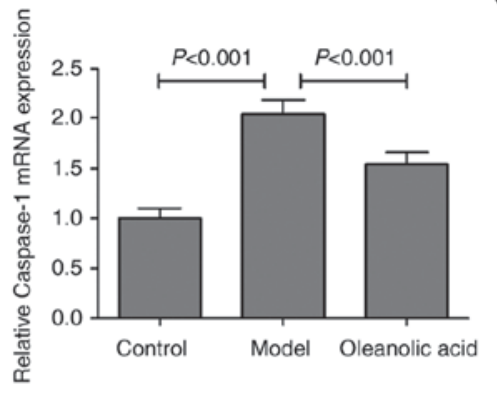

E
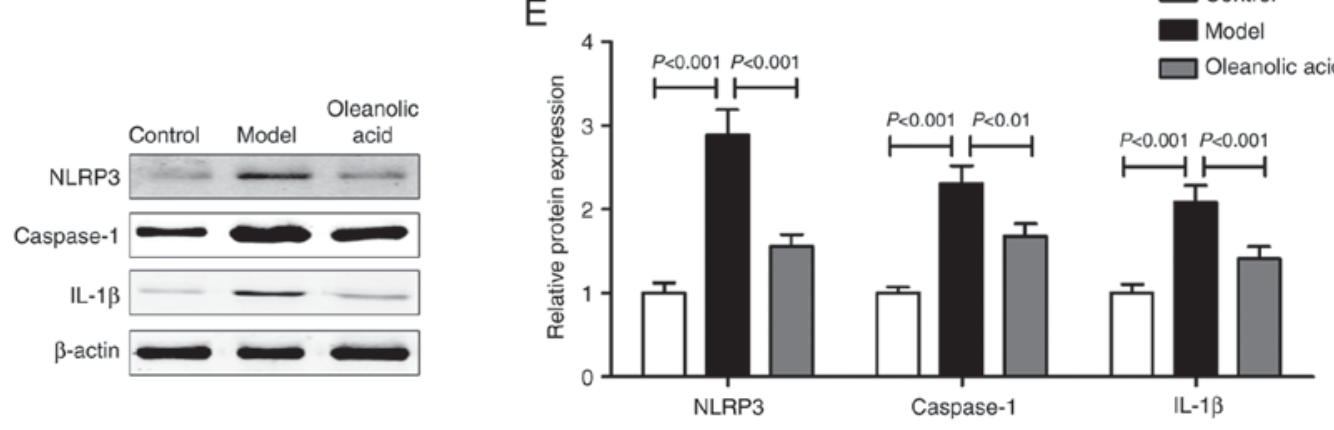

C

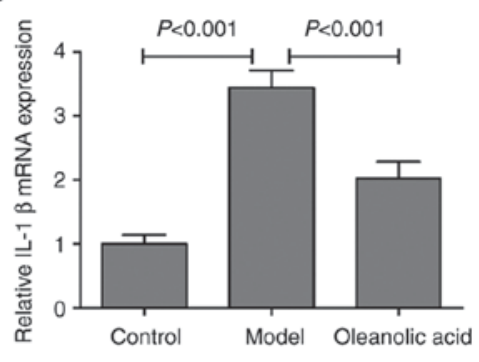

$\square$ Control

Model

$\square$ Oleanolic acid

NLRP3

Figure 6. Oleanolic acid inhibits the expression of NLRP3 inflammasome components. Normal rats and streptozotocin-induced diabetic rats were fed with or without oleanolic acid (100 mg/kg/day) between weeks 6 and 12 . The mRNA expression levels of (A) NLRP3, (B) caspase-1 and (C) IL-1 $\beta$ in the carotid artery were measured using reverse transcription-quantitative polymerase chain reaction analysis. (D and E) protein expression levels of NLRP3, caspase-1 and IL-1 $\beta$ in the carotid artery were measured using western blot analysis. Values are expressed as the mean \pm standard error of the mean ( $n=6$ in each group). NLRP3, nucleotide-binding domain, leucine-rich-containing family, pyrin domain-containing-3; IL, interleukin.

results suggested a major role for oleanolic acid in reducing the circulating levels of pro-inflammatory cytokines. To further investigate the anti-inflammatory effect of oleanolic acid and its molecular mechanisms in the progression of hyperglycemia-induced carotid artery injury, the expression levels of NLRP3 inflammasome components were examined in the carotid arteries of the rats. RT-qPCR and western blot analyses of the expression levels of NLRP3 inflammasome components in the carotid arteries revealed that the mRNA levels of NLRP3 (Fig. 6A), caspase-1 (Fig. 6B) and IL-1 $\beta$ (Fig. 6C), and their protein levels (Fig. 6D and E) were higher in the diabetic rats, compared with those in the control group rats. Oleanolic acid administration led to significant decreases in the levels of these NLRP3 inflammasome components. 


\section{Discussion}

Inflammation cytokines are crucial in the initiation and progression of vascular diseases. The NLRP3 inflammasomes have recently emerged as a pivotal regulator of the chronic inflammatory response (23), however, the expression of NLRP3 inflammasomes in carotid artery injury have not been fully elucidated. In the present study, NLRP3 inflammasome components were significantly upregulated in the carotid artery of diabetic rats. Oleanolic acid was closely linked to these factors and was able to reverse the hyperglycemia-induced upregulation of NLRP3 inflammasome components in the diabetic rats.

A previous study indicated that NLRP3 inflammasome components, including NLRP3, ASC, caspase-1, IL-1 $\beta$ and IL-18, are expressed at high levels in human carotid atherosclerotic plaques (8). In addition, NLRP3 is overexpressed in the aorta of patients with coronary atherosclerosis, and the aortic expression of NLRP3 is correlated with the severity of coronary artery disease and atherosclerotic risk factors (9). There is a positive correlation between the NLRP3 inflammasome and cytokine levels of IL-1 $\beta$ and IL-18 (23). NLRP3 forms a multiprotein complex, termed the inflammasome, which acts as a platform for activation of the cysteine protease caspase-1. Pro-forms of proinflammatory cytokines IL-1 $\beta$ and IL-18 are then cleaved by active caspase-1 to their active forms (9). Previous studies suggest a link between inflammation and glucose metabolic disturbance-associated diseases, including diabetes and atherosclerosis $(24,25)$. The results of the present study revealed a significant increase in the serum levels of TNF- $\alpha$, IL- $1 \beta$, IL- 6 and IL-18 in a rat model of carotid artery injury. As expected, the RT-qPCR and western blot analyses showed a significant upregulation in the expression levels of NLRP3, caspase- 1 and IL- $1 \beta$ in the carotid artery of diabetic rats. In apolipoprotein E-knockout $\left(\mathrm{ApoE}^{-/-}\right)$or low-density lipoprotein receptor-knockout mice, the deletion of IL-1 $\beta$ leads to a decrease in the size of atherosclerotic lesions $(9,26)$. Similar findings have been observed in ApoE ${ }^{-/} /$caspase $^{-1 /}$ double knockout or ApoE ${ }^{-1 /} / \mathrm{IL}^{-18^{-/}}$double knockout mice $(27,28)$. These results suggest that the NLRP3 inflammasome signaling pathway is important in cardiovascular diseases.

Several studies have demonstrated that oleanolic acid has a positive effect on diabetes-associated complications $(13,14)$, however, the underlying mechanisms remain to be fully elucidated. Several studies have demonstrated the ability of oleanolic acid to normalize blood glucose levels in rodents with diet-induced obesity or diabetes through the regulation of glucose 6 phosphate and forkhead box protein $\mathrm{O} 1$ in the liver $(15,29)$. Consistent with these results, the present study showed that oleanolic acid administration improved hyperglycemia-induced body weight and glucose metabolic disorders in the rats with carotid artery injury. Feeding an HFD or hyperglycemia leads to an accentuated proinflammatory state in local tissues and peripheral circulation (15). The inflammation of local tissues contributes to hyperglycemia, insulin resistance and diabetes-associated complications (30). Previous studies have confirmed that oleanolic acid inhibits inflammation through the suppression of NF- $\mathrm{KB}$ signaling, the inhibition of cytokines, including IL- 6 and TNF- $\alpha$, and the increased production of antioxidants via the promotion of $\mathrm{Nrf} 2$ signaling $(15,30)$. In the present study, it was demonstrated that oleanolic acid improved stenosis and neointimal hyperplasia of the carotid artery in diabetic rats. In addition, oleanolic acid significantly prevented hyperglycemia-induced vascular endothelial dysfunction in the diabetic rats. Oleanolic acid also inhibited the mRNA and protein expression levels of NLRP3, caspase-1 and IL-1 $\beta$ in the rates with hyperglycemia-induced carotid artery injury. These data provide in vivo evidence that oleanolic acid is an effective NLRP3 inflammasome inhibitor, which leads to the alleviation of carotid artery injury in diabetic rats.

In conclusion, the present study revealed a novel triggering mechanism of oleanolic acid in diabetic rats with carotid artery injury. The underlying mechanism was mediated, at least partially, through the suppression of NLRP3 inflammasome signaling pathways, the involvement of which may be an early event leading to neointimal hyperplasia and endothelial dysfunction.

\section{References}

1. Irie Y,Katakami N,KanetoH,KasamiR, SumitsujiS, YamasakiK, Tachibana K, Kuroda T, Sakamoto K, Umayahara Y, et al: Maximum carotid intima-media thickness improves the prediction ability of coronary artery stenosis in type 2 diabetic patients without history of coronary artery disease. Atherosclerosis 221: 438-444, 2012.

2. Tian F, Chen Y, Liu H, Zhang T, Guo J and Jin Q: Assessment of characteristics of neointimal hyperplasia after drug-eluting stent implantation in patients with diabetes mellitus: An optical coherence tomography analysis. Cardiology 128: 34-40, 2014.

3. Yang J, Fan Z, Yang J, Ding J, Yang C and Chen L: MicroRNA-24 attenuates neointimal hyperplasia in the diabetic rat carotid artery injury model by inhibiting Wnt 4 signaling pathway. Int J Mol Sci 17: E765, 2016.

4. Wang K, Zhou Z, Zhang M, Fan L, Forudi F, Zhou X, Qu W, Lincoff AM, Schmidt AM, Topol EJ and Penn MS: Peroxisome proliferator-activated receptor gamma down-regulates receptor for advanced glycation end products and inhibits smooth muscle cell proliferation in a diabetic and nondiabetic rat carotid artery injury model. J Pharmacol Exp Ther 317: 37-43, 2006.

5. Simon DI: Inflammation and vascular injury: Basic discovery to drug development. Circ J 76: 1811-1818, 2012.

6. Sirico G, Spadera L, De Laurentis M and Brevetti G: Carotid artery disease and stroke in patients with peripheral arterial disease. The role of inflammation. Monaldi Arch Chest Dis 72: 10-17, 2009.

7. Xia M, Boini KM, Abais JM, Xu M, Zhang Y and Li PL: Endothelial NLRP3 inflammasome activation and enhanced neointima formation in mice by adipokine visfatin. Am J Pathol 184: 1617-1628, 2014.

8. Shi X, Xie WL, Kong WW, Chen D and Qu P: Expression of the NLRP3 Inflammasome in Carotid Atherosclerosis. J Stroke Cerebrovasc Dis 24: 2455-2466, 2015.

9. Zheng F, Xing S, Gong Z and Xing Q: NLRP3 inflammasomes show high expression in aorta of patients with atherosclerosis. Heart Lung Circ 22: 746-750, 2013.

10. Goyal SN, Mahajan UB, Chandrayan G, Kumawat VS, Kamble S, Patil P, Agrawal YO, Patil CR and Ojha S: Protective effect of oleanolic acid on oxidative injury and cellular abnormalities in doxorubicin induced cardiac toxicity in rats. Am J Transl Res 8: 60-69, 2016.

11. Madlala HP, Van Heerden FR, Mubagwa K and Musabayane CT: Changes in renal function and oxidative status associated with the hypotensive effects of oleanolic acid and related synthetic derivatives in experimental animals. PLoS One 10: e0128192, 2015.

12. Yu Z, Sun W, Peng W, Yu R, Li G and Jiang T: Pharmacokinetics in vitro and in vivo of two novel prodrugs of oleanolic acid in rats and its hepatoprotective effects against liver injury induced by CC14. Mol Pharm 13: 1699-1710, 2016. 
13. Liese J, Abhari BA and Fulda S: Smac mimetic and oleanolic acid synergize to induce cell death in human hepatocellular carcinoma cells. Cancer Lett 365: 47-56, 2015.

14. Wang X, Chen Y, Abdelkader D and Hassan W: Combination therapy with oleanolic acid and metformin as a synergistic treatment for diabetes. J Diabetes Res 2015: 973287, 2015.

15. Camer D, Yu Y, Szabo A and Huang XF: The molecular mechanisms underpinning the therapeutic properties of oleanolic acid its isomer and derivatives for type 2 diabetes and associated complications. Mol Nutr Food Res 58: 1750-1759, 2014

16. Lu J, Wu DM, Zheng YL, Hu B, Cheng W, Zhang ZF and Shan Q: Ursolic acid improves high fat diet-induced cognitive impairments by blocking endoplasmic reticulum stress and IkappaB kinase beta/nuclear factor- $\kappa \mathrm{B}$-mediated inflammatory pathways in mice. Brain Behav Immun 25: 1658-1667, 2011

17. Buus NH, Hansson NC, Rodriguez-Rodriguez R, Stankevicius E, Andersen MR and Simonsen U: Antiatherogenic effects of oleanolic acid in apolipoprotein E knockout mice. Eur J Pharmacol 670: 519-526, 2011.

18. Zheng Z, Chen H, Wang H, Ke B, Zheng B, Li Q, Li P, Su L, Gu Q and $\mathrm{Xu} \mathrm{X}$ : Improvement of retinal vascular injury in diabetic rats by statins is associated with the inhibition of mitochondrial reactive oxygen species pathway mediated by peroxisome proliferator-activated receptor gamma coactivator 1alpha. Diabetes 59: 2315-2325, 2010

19. Chai J, He Y, Cai SY, Jiang Z, Wang H, Li Q, Chen L, Peng Z, $\mathrm{He} \mathrm{X}, \mathrm{Wu} \mathrm{X}$, et al: Elevated hepatic multidrug resistance-associated protein 3/ATP-binding cassette subfamily $\mathrm{C} 3$ expression in human obstructive cholestasis is mediated through tumor necrosis factor alpha and c-Jun NH2-terminal kinase/stress-activated protein kinase-signaling pathway. Hepatology 55: 1485-1494, 2012.

20. Dushpanova A, Agostini S, Ciofini E, Cabiati M, Casieri V, Matteucci M, Del Ry S, Clerico A, Berti S and Lionetti V: Gene silencing of endothelial von Willebrand factor attenuates angiotensin II-induced endothelin-1 expression in porcine aortic endothelial cells. Sci Rep 6: 30048, 2016.

21. Tousoulis D, Kampoli AM, Tentolouris C, Papageorgiou N and Stefanadis C: The role of nitric oxide on endothelial function. Curr Vasc Pharmacol 10: 4-18, 2012.
22. Wong BW, Wong D, Luo $\mathrm{H}$ and McManus BM: Vascular endothelial growth factor-D is overexpressed in human cardiac allograft vasculopathy and diabetic atherosclerosis and induces endothelial permeability to low-density lipoproteins in vitro. J Heart Lung Transplant 30: 955-962, 2011.

23. Satoh M, Tabuchi T, Itoh T and Nakamura M: NLRP3 inflammasome activation in coronary artery disease: Results from prospective and randomized study of treatment with atorvastatin or rosuvastatin. Clin Sci (Lond) 126: 233-241, 2014.

24. Saita D, Ferrarese R, Foglieni C, Esposito A, Canu T, Perani L, Ceresola ER, Visconti L, Burioni R, Clementi M and Canducci F: Adaptive immunity against gut microbiota enhances apoE-mediated immune regulation and reduces atherosclerosis and western-diet-related inflammation. Sci Rep 6: 29353, 2016.

25. Chow BS, Koulis C, Krishnaswamy P, Steckelings UM, Unger T, Cooper ME, Jandeleit-Dahm KA and Allen TJ: The angiotensin II type 2 receptor agonist compound 21 is protective in experimental diabetes-associated atherosclerosis. Diabetologia 59: 1778-1790, 2016

26. Kirii H, Niwa T, Yamada Y, Wada H, Saito K, Iwakura Y, Asano M, Moriwaki H and Seishima M: Lack of interleukin-1beta decreases the severity of atherosclerosis in ApoE-deficient mice. Arterioscler Thromb Vasc Biol 23: 656-660, 2003

27. Mallat Z, Corbaz A, Scoazec A, Graber P, Alouani S, Esposito B, Humbert Y, Chvatchko Y and Tedgui A: Interleukin-18/interleukin-18 binding protein signaling modulates atherosclerotic lesion development and stability. Circ Res 89: E41-E45, 2001.

28. Gage J, Hasu M, Thabet M and Whitman SC: Caspase-1 deficiency decreases atherosclerosis in apolipoprotein E-null mice. Can J Cardiol 28: 222-229, 2012.

29. Gao D, Li Q, Li Y, Liu Z, Fan Y, Liu Z, Zhao H, Li J and Han Z: Antidiabetic and antioxidant effects of oleanolic acid from Ligustrum lucidum Ait in alloxan-induced diabetic rats. Phytother Res 23: 1257-1262, 2009.

30. Osborn $\mathrm{O}$ and Olefsky JM: The cellular and signaling networks linking the immune system and metabolism in disease. Nat Med 18: 363-374, 2012. 